\title{
Psicoterapia breve en grupo: una modalidad práctica con enfoque constructivista y ecosistémico para la solución de problemas $^{*}$
}

\section{Brief groups psychotherapy: a practical embodiment with constructivist and ecosystemic for troubleshooting}

\author{
Mireya Medina Peralta** \\ Pontificia Universidad Javeriana, Colombia \\ Recibido: 19 de noviembre 2013 \\ Revisado: 20 de diciembre 2013 \\ Aceptado: 1 de febrero de 2014
}

\section{Resumen}

En el presente artículo se expondrá cómo se construyeron escenarios interventivos haciendo uso de la modalidad de psicoterapia breve en grupo con enfoque constructivista y ecosistémico, y cómo desde dichos escenarios se vehiculizaron, a través de las conversaciones generativas, nuevas formas de comprender las múltiples realidades y las diferencias que subyacen a estas. Durante el proceso, participaron cuatro consultantes y un psicoterapeuta (como centro mediador-posibilitador). Metodológicamente, se hizo uso de la modelización sistémica, en la cual el terapeuta, a partir de su capacidad y creatividad para generar nuevos niveles de comprensión de las realidades, fue moldeando escenarios de intervención en donde el motivo de consulta se transformó en el pretexto para generar cambios de orden lógico en los niveles de comprensión de cada uno de los participantes. Una de las estrategias utilizadas por el terapeuta fue el uso del correo electrónico narrativo, el cual hace referencia a una plataforma en la que los participantes del proceso consignaban narrativas escritas que permitían relatar los eventos emergentes durante el proceso y potencializar los cambios. Lo anterior facilitó la emergencia de soluciones a los diferentes problemas. 
Palabras clave: escenarios conversacionales, prácticas dialógicas, cambio de orden lógico, monitoreo psicoterapéutico, modelización sistémica, correo electrónico narrativo.

\section{Abstract}

This article will outline how interventional scenarios were constructed using the method of brief group psychotherapy through a systemic eco-constructivist approach and how new ways of understanding multiple realities and differences that underlie them arose through generative conversations directed in these scenarios. Four consultants and a psychotherapist (as mediator enablercenter) were involved in the process. Methodologically, systemic modeling, in which the therapist, drawing from his own abilities and creativity in order to generate new levels of understanding of the multiple realities, was able to shape intervention scenarios where the reason for consultation became the pretext for generating logical order changes in the levels of understanding of each of the participants. One of the strategies used by the therapist was the use of e-mail narrative, which refers to a platform on which participants in the process logged written narratives that enabled them to recount events emerging during the process and thus allowed them to enhance change. This facilitated the emergence of solutions to different problems.

Key words: Brief group psychotherapy, changing logical order, psychotherapeutic monitoring systemic modeling, e-mail narrative.

\section{Introducción}

Cuando un consultante ve lo que no había visto, tanto él como la terapia se llenan de ventanas. La terapia se transforma en una especie de espejo que devela, de manera casi inverosímil, la luz emergente de una entramada de hilos que provienen de recursos y posibilidades inéditas en los consultantes.

La realidad del consultante podría asemejarse a un globo sin aire, al cual se le trazan diferentes líneas que parecen muy unidas entre sí, sin mayor espacio entre unas y otras; sin embargo, al inflarlo se desdoblan múltiples espacios que no eran percibidos anteriormente y que otorgan posibilidades de acción. En relación con lo anterior, el modelo que se considera fundamental construir es el de un escenario de grupo generativo para poder intervenir sobre los motivos de consulta de cuatro estudiantes javerianos. De esta manera, se busca acceder a la comprensión sobre los efectos de la implementación de esta modalidad en el Centro de Asesoría Psicológica de la Pontificia Universidad Javeriana.

\section{Distinciones teóricas}

El desarrollo teórico parte de autores que procuran el cambio a través del enfoque ecosistémico y complejo; ellos, en las últimas décadas, han considerado como parte esencial del trabajo terapéutico la necesidad de complejizar los procedimientos para la intervención en los problemas humanos. Se considera lo humano como una emergencia que se engendra en la interdependencia creadora entre lo cultural, lo subjetivo, lo cerebral y lo biológico. Por tanto, su objeto de estudio opera en la ritualización de la interacción y la atribución de significado a lo vivido en los operadores espaciotemporales de los vínculos. En coherencia con lo afirmado, los motivos de consulta se visualizaron como interferencias a la autonomía de los sujetos y a su proceso de individuación en un contexto eco-dependiente, los cuales emergen en determinadas configuraciones relacionales.

En este sentido, diferentes teóricos como Bateson (1993), Foerster (1994), Anderson (1997), Watzlawick (1993), et al. consideran que los pro- 
blemas y sus soluciones se generan en la relación y cobran sentido en la interacción y en los contextos relacionales -puestos en escena a través de las conversaciones interventivas-, por lo cual el modelo de intervención se empeñó en desarrollar marcos diferentes, opuestos al uso tradicional de una comunicación que implica una causalidad lineal y específicamente monológica de las conversaciones. En este sentido, se conversó con cuatro participantes en un contexto grupal de carácter terapéutico que abordó los motivos de consulta como una realidad ecosistémica. En esta última tuvieron cabida las reflexiones de los participantes y el psicoterapeuta en torno a las teorías que explicaron estos motivos (lo deductivo), procederes que los postularon (lo inductivo) y particularidades que los enriquecieron (abductivo) y que facilitaron emergencias relacionales, consensuales y contextuales.

Se animaron formas complejas de pensar el fenómeno como, por ejemplo, la visión de la eco-etoantropología de Miermont (1987), que constituye una teoría abierta bajo los principios de la autoeco-organización. Bajo estos se considera que los eventos se reproducen a través del espíritu, en coherencia con las condiciones cerebrales -y también culturales- favorables, creando y recreando las realidades que nos construyen al nosotros construirlas. Se dialogó de manera recursiva y dialógica con el grupo de consultantes para movilizar, dinamizar y conectar con el contexto formas de solucionar sus dificultades bajo los principios de observación y emergencia, esto al otorgar al lenguaje y a las conversaciones un carácter de facilitadoras y dinamizadoras de lo humano dentro del contexto del vínculo como principio explicativo de los problemas humanos.

\section{Método}

\section{Tipo de estudio}

El presente estudio se realizó dentro de los parámetros de investigación-intervención que constituyó un ejercicio reflexivo en el orden de la modelización sistémica (Hernández, 2004), lo cual implicó que el psicoterapeuta construyera con el modelo transformador, el cual, a su vez, le trans- formó bajo el principio de observación emergente en la construcción.

Se configuró un modelo particular y flexible a las hipótesis emergentes para conectarlas con el contexto y la posibilidad de cambio bajo una metodología cualitativa, por ejemplo, con Sabino (1978) se recurre a la creatividad del investigador para adaptar las metodologías disponibles al abordaje de la problemática.

\section{Fuentes de recolección de información}

- Sesiones transcritas con los participantes

- Protocolos de las sesiones

- Correos electrónicos terapéuticos

- Reflexiones de los participantes

\section{Niveles de observación}

Cuatro miembros de la comunidad universitaria con un motivo de consulta que participaron en la convocatoria bajo el título "yo, tú, él, nosotros [...] diálogos que construyen soluciones": psicoterapeuta. Investigador-interventor.

\section{Instrumentos}

- Formatos de transcripción

- Matrices

- Correos electrónicos

- Entrevistas semiestructuradas

\section{Procedimiento}

- Fase 1. Construcción del contexto psicoterapéutico

El equipo se sienta en un círculo y se abre la sesión definiéndola como un espacio para escuchar las voces del grupo. Se hace un resumen de la historia de la reunión y de las expectativas del equipo; se inicia preguntando cómo quisieran utilizar el tiempo y quién cree que debe empezar. Se explican algunos de los principios que organizan las conversaciones sin una idea específicamente 
preconcebida sobre los temas que deben debatirse de manera abierta. Los terapeutas tienen la responsabilidad de hacer preguntas de orden recursivo y dialógico (Seikkula, 2005).

\section{- Fase 2. Escenarios conversacionales}

Las distinciones operativas que organizaron los diálogos estuvieron delimitados por los modelos y emergencias del ámbito de intervención psicoterapéutica, es decir, una relación especifica: psicoterapeuta-consultantes, reunidos con fines e intereses de cambio en el contexto de un discurso confidencial, en una intervención en grupo en el contexto del Centro de Asesoría Psicológica. Se utilizó como plataforma de acceso a seis escenarios conversacionales de orden recursivo y dialógico, cada uno con dos horas de duración semanales.

- Fase 3. Análisis de resultados

Se utilizó el análisis de narrativas, que implicó visualizar la manera en que el lenguaje construye realidades y nuevos entendimientos de mundo. En este sentido, se siguen los parámetros de Sabino (1978), que sugieren una postura hermenéutica interpretativa para las prácticas discursivas que son contadas, recontadas y vueltas a contar considerándolas en las dimensiones tiempo y espacio correspondientes.

El estudio es coherente con la Resolución 008430 de 1993 del Ministerio de Salud; por tal motivo, la confidencialidad de los participantes, las grabaciones, y el consentimiento informado son principios fundamentales. De esta manera, la intervención prioriza el componente interventivo que busca la generación de soluciones en los participantes a partir de la práctica discursiva dentro del contexto terapéutico.

\section{Participantes}

Un psicólogo y cuatro miembros de la comunidad javeriana.

\section{Pregunta de investigación}

¿Cuáles son los efectos de una modalidad de psicoterapia breve en grupo implementada desde una perspectiva constructivista ecosistémica utilizando las conversaciones reflexivas en el orden recursivo y dialógico?

\section{Objetivo de investigación}

Comprender los efectos de la implementación de esta modalidad en los niveles de comprensión de los participantes y en la emergencia de posibilidades que aportan a la construcción de soluciones posibilitadoras de acciones de cambio en sentido positivo. Se considera la experticia del terapeuta para traer información de orden deductivo, inductivo y abductivo con un grupo de participantes que consultan por diferentes motivos.

\section{Categorías de análisis}

En el orden de la modelización sistémica, las categorías predeterminadas fueron:

Categoría 1. Modo de operar psicoterapéutico: prácticas dialógicas y conversaciones generativas que desde una perspectiva eco sistémica facilitaron al psicoterapeuta vehiculizar conexiones deductivas, inductivas y abductivas; a partir de la pericia de los consultantes para construir posibilidades de manera dirigida (Shotter, 2011; Seikkula, 2010).

Categoría 2. Cambios de orden lógico en los niveles comprensivos de los participantes: comprensión particularizada de la experiencia de los consultantes que se logró a través de un proceso de ajuste y reajuste de la visión de los consultantes para enfocar diferentes facetas y posibilidades de la realidad que preocupaba como puente para la emergencia de soluciones (Bateson, 1993; Schitmann, 1995).

Categoría 3. Construcción y modelización del escenario conversacional: se refiere a discursos reflexivos que retroalimentan los procederes del grupo de participantes y del psicoterapeuta frente al cómo se está construyendo en los tejidos relacionales la práctica de las conversaciones generativas para soluciones posibles (Packman, 1995). 
Categoría 4. Resiliencia: la capacidad del ser humano de sobreponerse a periodos de dolor o traumas; esto de forma tal que pueda reconstruirse, repararse -sin que la vida quede truncada por ello- a través de cambios en las maneras de significar, de relatar y de actuar en su funcionamiento interaccional para contrarrestar los efectos no deseados de la dificultad (Cyrulnik, 2009).

\section{Procedimiento de intervención}

\section{Construcción del contexto interventivo-investigativo}

En el Centro de Asesoría Psicológica se accede al servicio por medio de un proceso de orientación al servicio, el cual, a través de una psicóloga, facilita que la persona ubique el servicio que requiere de acuerdo con su necesidad. En este contexto se les ofreció la posibilidad de participar en el grupo de intervención.

1. Invitación: se invitó a cuatro miembros de la comunidad universitaria javeriana que tenían un motivo de consulta, a inscribirse a un grupo de intervención coordinado por un psicoterapeuta bajo el título: "yo, tú, él, nosotros [...] diálogos que construyen soluciones".

Se motivó la creatividad para presentar su requerimiento de consulta en la primera sesión, de manera tal que estuviese "iluminado" por la historia de vida, puesto que se consideró que desde el inicio de la recepción era importante procurar una descripción más allá del motivo de consulta.

Se les explicó que el psicoterapeuta no solo sería un receptor de comunicación sino que también iba a operar con la intención de crear un escenario generativo alrededor de los problemas; esto les permitió participar, de manera dialógica y recursiva, guiados por el psicoterapeuta. De este modo, se desorganizó la realidad para organizarla de otra manera, conectando datos y pesquisas, intuiciones; reconociendo lo ambiguo, lo aleatorio, lo incierto y lo cierto, para poder acceder a una construcción de significados y acciones de mayor complejidad contextual.
2. El grupo de intervención: estuvo conformado por cuatro estudiantes: María (M), de 20 años de edad; Esperanza (E), de 23 años; Pedro (P), de 22 años, y Juan (J), de 22 años. Se citó a los participantes a seis sesiones durante dos horas semanales. Se necesitó de un salón sencillo, colores, plastilina, lápiz, papel; café o agua aromática.

3. Procedimiento de intervención: ¿cómo se inició? El equipo se sentó en un círculo y se abrió la sesión definiéndola como un espacio para escuchar las voces de los integrantes del grupo con respecto a los motivos de consulta y generar conversaciones posibilitadoras.

¿Con qué llegaron? Los participantes llegaron con una visión rigidificada del problema, es decir, con un primer análisis de lo que les ocurría y de cómo ocurría lo que ocurría: con una percepción en blanco y negro, fija y repetitiva:

M: Creo que no está bien, él tuvo el accidente conmigo [...], y ahora yo no puedo dejarlo [...], sería muy desgraciada si lo hiciera.

E: Yo soy así [...]. No soy bonita [...], soy más bien feíta [...], no tengo novio [...], siempre me dejan y eso que me portó bien y soy muy detallista, muy tierna... no puedo ser diferente [...], les llamo y estoy pendiente de ellos [...], y no de mi tesis [...], no me puedo concentrar.

P: Soy el menor de la familia [...]. Siempre fuimos muy unidos, yo era el consejero... les cuidaba los bebés a mis hermanos [...], le ayudaba a mi mamá [...], ella no quiere contratar empleada porque mi papá se enamoraría de la empleada.

J: Esta enfermedad hizo que mis papás me cuidaran todo el tiempo, posponer los estudios [...], no aprendí a hacer amigos y ahora prefiero no venir a clase, pues no sé hacer amigos.

El terapeuta $(\mathrm{T})$ inicialmente configuró la intervención de manera que el flujo de conversaciones grupales se tejiera con las historias de cada participante en sentido recursivo y generativo: 
T: ¿fue difícil para todos retar la culpa, el pesar o el reproche? ¡Qué coincidencia! ¿Todos ustedes se han sentido en algún momento ubicados en el rol de víctima? ¿Estarían dispuestos a iniciar una película de desafío a estos tigres de papel que les sometían?

La reflexión en torno a estas preguntas posibilitó a los participantes ubicarse como espectadores y protagonistas en el grupo; sensibilizarse al efecto paralizante que estas realidades causaban a sus escenarios de vida, así como la manera en que ellos mismos se disponían a mantener vigentes las historias que les paralizaban. Posteriormente, se propusieron las reglas de diálogo generativo como ruta para encontrar posibilidades novedosas que les permitieran enfrentar las condiciones que generan la dificultad.

¿Cuáles fueron los criterios que organizaron las conversaciones? Desde el inicio mismo de las conversaciones se establece un proceso conjunto en el que se incluyen, de manera compleja, variables de orden dialógico que dan valor a las visiones particularizadas, provenientes del interjuego reflexivo del terapeuta con los consultantes. Lo anterior se encuentra sustentado en la teoría binocular del cambio y la lógica abductiva sugerida por Bateson (1993), en la que se entretejen los aportes provenientes de una lógica deductiva e inductiva.

Esta práctica interventiva exigió al terapeuta una actitud alerta y a la deriva con las historias de los participantes, pues compartían experiencias profundas que fueron conectando cada vez más a todas las personas involucradas; estas se tornaron coautoras de la historia que "rerelataban" en un proceso de legitimización del otro y de sí mismo (Maturana, 1976).

Al mismo tiempo, se consideró conveniente el uso de medios narrativos escritos con fines terapéuticos, como marco de una inteligibilidad que brindaría un contexto para fortalecer las posibilidades construidas desde las versiones de vida posibilitadoras, los relatos alterados o las atribuciones de significados novedosos y el funcionamiento interaccional suscitado con sentido de resiliencia individual-relacional y de acción ecológica.
El psicoterapeuta envió semanalmente a los consultantes del grupo correos electrónicos que organizaron, exaltaron o invocaron semióticamente algunos de los sub-relatos que, según el punto de vista del psicoterapeuta, requerían mediación activa para vincular ideas que especificaran, prescribieran o reforzaran posibilidades de funcionamiento interaccional y de resiliencia individual relacional. Estos fueron enviados después de cada escenario conversacional.

\section{Resultados}

¿Cuáles fueron los procesos interactivos demarcados en los niveles de reflexión y conciencia que coexistían con los motivos de consulta de los participantes? Estos niveles constituyeron un primer acercamiento y un pretexto para nombrar el grupo como uno que está en todos y un todo que está en uno. Esto permitió identificar y subrayar coincidencias, diferencias e interacciones entre las acciones grupales e individuales, entre sus concepciones, los dispositivos internos que las inician y los ambientes naturales-artificiales que les dan sentido. Tal y como se muestra en la figura 1 , se puede visibilizar el interjuego de los procesos históricos, emocionales y psicológicos de los participantes puestos en el contexto de intervención.

\section{Momentos interventivos}

El proceso interventivo que se presentará a continuación reúne de manera general las estrategias y técnicas de intervención que no alcanzan a proyectar la riqueza de las conversaciones, y menos aún la complejidad interactiva en la transformación de los participantes. Es importante señalar que las estrategias de intervención fueron emergiendo en el fluir de las conversaciones; en coherencia con el modelo de las prácticas dialógicas que sugieren de la flexibilidad y evitan una planeación predeterminada de las estrategias interventivas. La figura 2 tiene como propósito condensar los diferentes momentos que, en forma recursiva y sin un orden establecido, emergieron durante el proceso de intervención. 
Figura 1. Procesos interactivos que coexistían con los motivos de consulta.

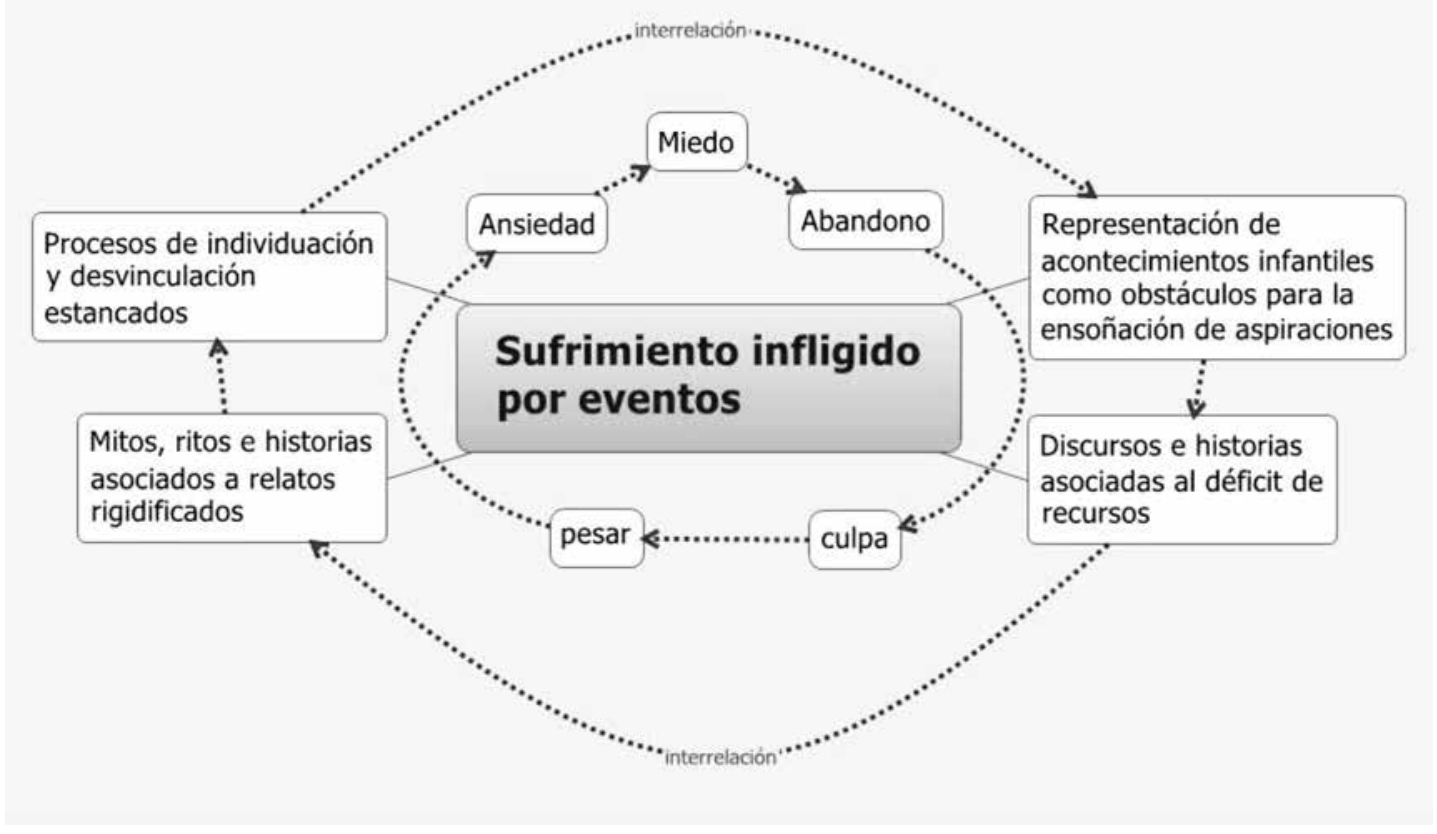

Fuente: elaboración propia.

\section{Correos electrónicos narrativos con fines interventivos}

A continuación se presentarán algunos de los contenidos de los correos electrónicos con su correspondiente intención terapéutica. Para facilitar su comprensión se realizó una tabla (tabla 1), en la que se condensan, por un lado, los correos electrónicos enviados por el psicoterapeuta, y por otro lado, la estrategia interventiva, es decir, la intención terapéutica con la cual se escribe lo enviado. Estas narrativas escritas y semióticamente organizadas por el psicoterapeuta continuaron brindando un marco alternativo, no solo para la atribución de significado sino también para ofrecer una fuente de autenticación reescrita de los eventos emergentes a nivel interaccional, lingüístico y sistémico. Al mismo tiempo, facilitaron un mayor compromiso del grupo como testigo presencial para contribuir al desarrollo y acción de las soluciones de los otros y de sí mismo. 
Figura 2. Momentos de la intervención.

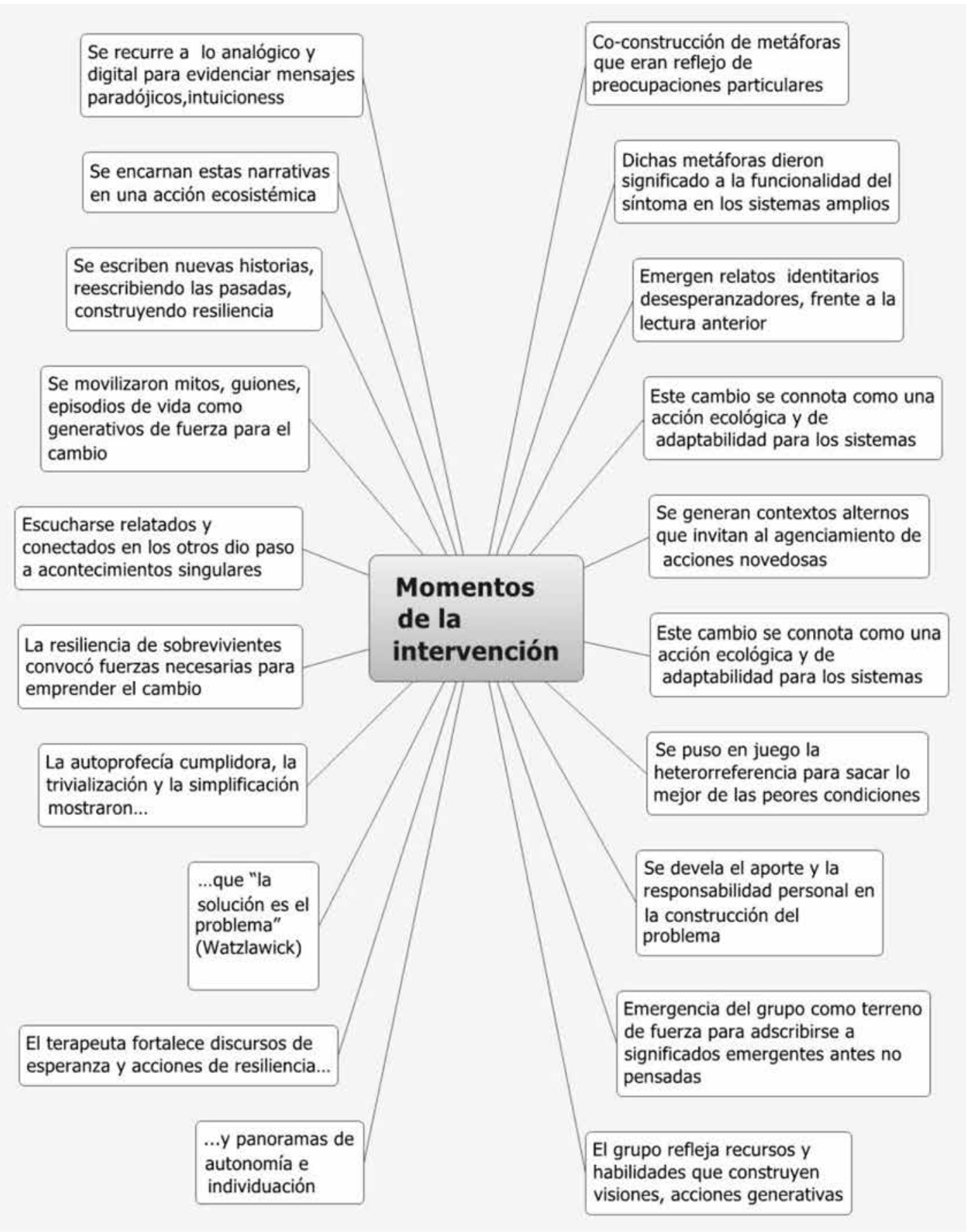

Fuente: elaboración propia. 
Tabla 1.

Correo electrónico como estrategia para centralizar aspectos emergentes.

Correo electrónico enviado por el psicoterapeuta a los consultantes

Creo que no me equivoco cuando utilizo la frase de Mejía, "duro de matar", para decirles que ustedes son: "duro de matar 10".

Yo estoy en absoluto acuerdo con $\mathrm{J}$ cuando refiere que $\mathrm{E}$, al igual que $\mathrm{J}$, puede ir más allá del hábito de actuar y evidenciar comportamientos atravesados por los lentes eternos de un episodio de abuso, y, que a mi entender, puede también decidir liberarse de este hábito $\mathrm{y}$ declarar la independencia de estos lentes.

Creo que se fue imponiendo, en J, tal fuerza interior, que detrás de la armadura apareció el caballero. Decidió no pagar el karma y esa decisión lo llevará por caminos de libertad que apenas empieza a conocer.

En la reunión de hoy se hizo evidente el hecho de que los miedos, la culpa y el pesar nos limitan, en la mayoría de los casos, nuestros escenarios de libertad, y como lo dijo $P$, venimos biológicamente para dar toda una gama de respuestas, y creo además que tenemos incidencia sobre la dirección que les demos.

Una pregunta interesante para ustedes es, ¿cómo lo lograrán? ¿Qué les facilitará tal fuerza? ¿Si continúan como van, cómo se visualizan en tres años? ¿Qué brújula van a construir para salir del laberinto? Si por alguna razón se regresaran, ¿cómo se las ingeniarán para rechazar una invitación de entrar en él, y cuál sería el mecanismo si por alguna razón volviesen a abrir esta puerta?

\section{Estrategia interventiva}

La conversación dialógica permitió recrear discursos identitarios a partir de significados implícitos que potencian la activación de metas y acciones de cambio.

Se hace una predicción (whitesoniana), que emerge de una práctica dialógica en la pretensión de favorecer una profecía autocumplidora.

$\mathrm{E}$, puede ahora asumir una responsabilidad con respecto a la manera en que la lectura del problema le empobrece su visión de vida para redireccionarse hacia lecturas y actos más satisfactorios.

Se desafió en el discurso una práctica de sometimiento al trauma que era coherente con las prácticas sociales, de tal manera que se logró "descongelar" un comportamiento esperado culturalmente como efecto del trauma y dirigir la mirada hacia soluciones que $\mathrm{E}$ encontró liberadoras.

Utilizando un lenguaje recursivo y dialógico se abren posibilidades inéditas que al ser "rerelatadas" en narrativas escritas generaron acciones olvidadas, como lo fue retar la culpa y asemejarla a un “'Dementor' que quitaba energía".

Esta carta animó a las personas a escribir con anticipación estrategias y modos de protegerse ante una recaída. Las preguntas estuvieron asociadas a facilitar que las soluciones que se construyeron como escenarios posibles de acción antes no pensadas se mantuvieran en el tiempo a pesar de una recaída si la hubiese.

Fuente: elaboración propia.

\section{Discusión}

\section{Modos de operar psicoterapéutico}

\section{- Intervención psicoterapéutica eco-sistémica}

Los puntos de vista para la generación de soluciones estuvieron guiados por modos de operar deductivos, inductivos y abductivos de los consultantes, interpenetradas con los puntos de vista del psicoterapeuta, lo cual facilitó hipótesis para explicar la construcción de las dificultades y posibilidades para su deconstrucción.
La percepción, la intuición, las conjeturas, y el sentido común aportaron a una racionalidad sistémica dirigida al sentido contextual, desde la perspectiva del observador y de la emergencia como novedad de construcción (Morin, 1991). Esto permitió direccionar la intención de cambio hacia la posibilidad de generar movimientos en los modos de construir vínculos para lograr transformarlos y así generar un desarrollo de posibilidades (O`Halon, 1999).

El psicoterapeuta incluyó las dimensiones empíricas y las simbólicas que iban emergiendo en 
el contexto de grupo, lo cual facilitó integrar los contenidos emocionales y las consideraciones de tipo racional para generar acoplamientos lingüísticos, estructurales y funcionales en las conversaciones terapéuticas.

Los canales reflexivos generativos, en torno al modelo de intervención en los escenarios conversacionales, convergieron con un despertar de la creatividad que, como diría Anderson (1997), favorecieron la co-construcción de un contexto sostenible para la emergencia de soluciones.

La inclusión reflexiva y proactiva facilitó aperturas inéditas a través del monitoreo responsivamente dialógico de las conversaciones con el otro, cosa que permitió no sólo encontrar, sino también reparar y recuperar acciones y síntesis desde las historias y los relatos que emergieron en el grupo, en un proceso que requería de un escenario éticamente construido (Foucault, 1999).

Las prácticas dialógicas dieron paso a la posibilidad de navegar en los eventos traumáticos de manera abierta, con curiosidad y motivación para explorar la situación sin el vendaje de la etiqueta médica, realidad que acercó cada vez más la transformación de experiencias de vida.

En el grupo, las dinámicas conversacionales permitieron alejarse de las polarizaciones semánticas y de representaciones limitantes (por ejemplo, tristeza versus felicidad o dolor versus placer) que constituían barreras mentales en los escenarios habitacionales tras un sufrimiento que se experimentaba.

Los correos electrónicos fueron herramientas que facilitaron que los consultantes siguieran no solo revisando su relación con el motivo de consulta, sino también posibilitó que generaran nuevas posibilidades interaccionales a partir de leerlos, releerlos, e incluso responderlos en el intervalo de tiempo que pasaba entre una y otra sesión,

Los correos electrónicos le facilitaron al psicoterapeuta centralizar las acciones emergentes en las conversaciones generativas y a los consultantes agenciarse como testigos activos en la construcción y el desarrollo de posibilidades de vida de estos. Los correos electrónicos invitaron a los consultantes a practicar un intercambio entre los momentos de toma de conciencia con respecto a las alternativas de acción en términos de sus ecologías de relación sistémica; reflexionaron sobre lo que les significaba actuar las soluciones posibles, los significados atribuidos y las consecuencias relacionales de manera que tuvieron efectos positivos para contrarrestar los motivos de consulta.

\section{- La experticia del psicoterapeuta}

Un espantapájaros, un espectro, se esfuerza por no pensar porque es demasiado doloroso construir un mundo íntimo plagado de representaciones atroces (...), pero basta que ese espantapájaros encuentre un hombre vivo que le insufle un alma para que el dolor de vivir vuelva a tentarlo (Cyrulnik, 2001).

Fue indispensable una postura de "responsividad responsable" (Shotter, 2011); por parte del psicoterapeuta, en unión con una actitud de reciprocidad en los consultantes. Fue importante que el terapeuta hubiese integrado en sí mismo y en la manera de acceder a las realidades del grupo en que intervino la mentalidad "analógica" (mitológica y simbólica), tanto como la lógica experimental (argumentativa y formal), lo que permitió complejizar y aportar a la construcción por parte del grupo de respuestas eficaces que aportaron a las soluciones.

Fueron importantes dos modos de implementar el cambio y la transformación. Por una parte, se involucró una fuerza yin que fue en sí misma amorosa, confiada, suave, y sutil, pero a la vez eficiente y cuidadosamente selectiva desde el punto de vista técnico. Por otra parte, una fuerza yang implicó una acción más vigorosa en sentido contrario de las acciones que saboteaban el cambio y que mostraron en determinados momentos los consultantes y el grupo.

Las limitaciones con respecto al lenguaje y la visión ecosistémica y de aspectos políticos, económicos y sociales por parte del psicoterapeuta se conversaron con los consultantes para disminuir los límites de la acción psicoterapéutica y utilizar la información lo más productivamente posible. 


\section{Cambios de orden lógico en los niveles comprensivos de los consultantes}

- Los consultantes crearon significados, reconstruyeron experiencias y pudieron adquirir nuevos sentidos sobre vivencias que consideraban "terribles".

- Refirieron que habían experimentado que el poder estaba en cambiar el "adentro", sus propias voces, para poder rechazar las invitaciones de participar en determinadas danzas relacionales conflictivas.

- Los consultantes movilizaron eventos traumáticos rigidificados en la linealidad del pasado como organizador del presente y del futuro.

- Se estableció una relación recíproca entre los participantes, de manera que se descubrieron como coautores de soluciones.

- Utilizaron la curiosidad como forma de poner en circulación nuevas perspectivas de vida.

- Se sintieron "pescadores" de recursos y posibilidades novedosas.

- "Confesar que habían vivido" les ayudó a verse en otros y con otros; esta sensación les dio sentido de pertenencia, compañía y motivación para el desarrollo de camino interior y de su incidencia en las relaciones que construían.

- Las conversaciones recursivas constituyeron un canal de información cogenerador de historias que producen nuevas grados de comprensión y de oportunidades.

- Las conversaciones generativas según los participantes fueron como "abrir la caja de Pandora” para que surgiera la esperanza.

\section{Resiliencia y escenarios de grupo terapéutico}

\section{Modelización del escenario terapéutico}

- Las formas de proceder instructivas y reflexivas que delimitaron el contexto de las conversaciones interventivas fueron reconstruidas y refor- muladas constantemente por aquellos que a su vez fueron trasformados por estas.

- Dichos eventos se constituyeron en un reto para el terapeuta, puesto que los diferentes participantes activos y observadores de sí mismos requirieron también que el terapeuta estuviese presente y responsivo.

- La construcción de un proceso reflexivo permitió la retroalimentación continua y favoreció la inclusión del participante en la estructuración permanente del contexto de intervención.

- Los integrantes se empoderaron en un proceso de autonomía y auto-organización en el que estaban siendo partícipes de la fabricación de soluciones, descubriéndose en el agenciamiento de aquello que habían solicitado que se les agenciara.

- El escenario facilitó una postura ecosistémica, en donde se involucraba, de manera participativa y asociativa, a los jóvenes para cocrear acciones eficientes y facilitar una mejor utilización de recursos.

- El correo electrónico terapéutico se develó como plataforma reflexiva que no solo logró un alto nivel de sintonía con los consultantes, sino que al mismo tiempo impactó los sentidos asociados a la actividad de lectoescritura; esto fue plenamente acogido.

- El correo electrónico se develó como fuente de reescritura por parte del psicoterapeuta sobre las posibilidades emergentes; se develó como territorio alternativo del grupo generador de complejidad en las alternativas de vida desarrolladas en las conversaciones, en las posibilidades desentendidas y en la imaginación y creatividad silenciada.

\section{Resiliencia}

- Los jóvenes crearon significados, reconstruyeron experiencias y pudieron adquirir nuevos sentidos sobre vivencias que consideraban "terribles", logrando ampliar alternativas con narrativas diferentes que les abrieron el camino para novedades en sus modos de percibir, de interpretar sus problemas y de afrontarlos. 
- Refirieron que habían experimentado que el poder estaba en cambiar el "adentro" -sus propias voces- para poder rechazar las invitaciones de participar en determinadas danzas relacionales conflictivas.

- El escenario terapéutico posibilitó un proceso de aprendizaje para el afrontamiento de situaciones difíciles futuras.

- Los consultantes participaron en la construcción de nuevas acciones y posibilidades a partir de la movilización de los eventos traumáticos rigidificados que organizaban de alguna manera el presente y el futuro en los sistemas que habitaban.

- Movilizar las representaciones de traumas, de historias y de dolores liberó una fuerza de motivación y de sentido para emprender acciones en sentido positivo del cambio.

- La hetero-referencia posibilitó aportes novedosos a los niveles de comprensión del grupo, que a su vez permitieron la circulación de nuevas posibilidades.

- La exploración activa de las preocupaciones de los consultantes permitió la dinamización de formas de proceder lingüísticas y del funcionamiento estructural-sistémico.

- Esta modalidad de intervención en grupo sacó a los consultantes de ese tejido reflexión en su interior y les ofreció la oportunidad de redescubrir y liberar su autonomía y su potencia inventiva en donde no se impusieron las realidades, sino que se generaron posibilidades de cambio en un lenguaje común con los participantes, posibilitando un contexto de libertad en la emergencia de leguajes alternos (Goolishian y Anderson, 2002).

Los correos electrónicos como medio narrativo introdujeron mensajes de esperanza, de motivación al logro y de posibilidades de deconstrucción de relatos dominantes asociados al sufrimiento experimentado frente al motivo de consulta.

\section{¿Con qué se fueron los participantes?}

- M: "estas conversaciones con el grupo me convirtieron en una mujer dispuesta a retar las creencias de mi familia: ahora puedo creer en mí. Sé que la culpa es mala consejera y que puedo desafiarla. Ahora me defino como una persona que no asume la responsabilidad de otros y trabajo en asumir la mía. Me siento protagonista del 'desafío a evitar asumir la responsabilidad de otros"”.

- E: "entendí que mi prisa por ser amada venía de que me sentía que era 'mercancía que no sirve' y me di cuenta que era una mujer bella con derecho a crecer. Inicié mi tesis con un tutor particular y por primera vez sé que es pensarse sin la etiqueta del abuso. Tengo una nueva visión sobre mí y sobre mi historia de vida, producto de compartir y escuchar la forma en que los otros la interpretan, con diferencias fuertes, que si uno se detiene a pensar, ahí estaban”.

- P: "el hecho de verme como la muchacha de mi familia me hizo reír pero en verdad ese era mi cargo; renuncié a él. En un momento me dio pesar con mis hermanos porque ellos iban ahora a ayudar a mamá, pero recordé que no somos responsables de lo que los otros hagan".

- J: "haber participado de estos diálogos me hizo dar cuenta de que estaba como en un arroyo dando vueltas y vueltas; llegué siendo torpe y ahora soy inexperto. $Y$ era cuestión de entrenarse para llegar a las niñas; despedí las vacaciones' que me tenían paralizado y empecé 'el desafío de incluirme en la facultad'”.

\section{Conclusiones}

Con esta investigación se logró la construcción activa del contexto de intervención, de miradas, de significados diversos y de acciones particulares construidas con la intención de cambio. En esta modalidad de intervención psicoterapéutica se asociaron la emergencia del conocimiento, la reflexión y la acción como procesos generativos de la diferencia que aportaron no solo a la realidad que se quería cambiar, sino también al contexto que rodeaba la manera en que se construía el cambio.

Este espacio implicó un prediseño de focos de observación por parte del equipo, donde en grupo los consultantes reconstruyeron sus experiencias 
de vida, deviniendo coautores de cada vivencia en particular, las que con facilidad se visualizaron como una posibilidad de ser reconstruidas como bio-psico-sociales y eco-sistémicas, y que desde allí permitieron develar puntos de encuentro para suscitar los procesos dialógicos y las posibilidades de acción.

La modalidad de intervención actuó como enlace y plataforma que, al mismo tiempo, facilitó la construcción de novedades y tejió la trama de estas visiones novedosas promoviendo la responsabilidad responsiva, la presencia inminente, la conexión profunda, la intuición, y el desarrollo de la lógica abductiva. Esto, a su vez, constituye una posición epistemológica que se extrapola a la experiencia vital de reconstruirse y de experienciarse.

Las acciones reconstruidas potenciaron la transformación de pautas de relación con los pares y familiares, así como una mayor disposición hacia el esfuerzo por el conocimiento y el desarrollo del camino interior, generando cambios en los relatos cristalizados y posibilitando la emergencia de narrativas alternas, en donde el problema para cada uno de los participantes se transformó, en palabras de White y Epson (1993), "en un capítulo de vida y no en toda la vida".

Las conversaciones dialógicas en el contexto de la modalidad de intervención suscitaron una acción de reciclaje permanente que despertó la creatividad dormida y las conexiones olvidadas, facilitando el acceso a señales e información novedosa que se integró en los modos de darse cuenta cómo construyen sus experiencias de vida.

Bajo los principios de la modalidad de intervención, los dolores, las experiencias del pasado que se habían anquilosado, que les quitaban energía y robaban capacidad de percepción, de aprendizaje y de cambio en los contextos presentes de interacción, dejaron de visualizarse como una fatalidad inevitable e inabordable, para visualizarse como una realidad con posibilidad de cambio.

El despliegue que suscitan los enlaces inéditos, las vías olvidadas y los caminos escondidos y los acoplamientos sistémicos y estructurales son po- sibilidades instrumentales que promueven aprendizajes relacionales; permiten que posibilite la administración eficiente de las diferencias hacia la construcción de una puerta para la emergencia de acciones conjuntas y del desarrollo de vínculos sociales de afrontamiento, reparación y recuperación dentro del contexto de acción ecológica y relacional de Morín.

Finamente, los escenarios conversacionales se construyeron considerando los procesos de hominización de la psicoterapeuta y los cuatro consultantes, se convocaron sus historias y mundos emocionales, tocados por el dolor, la preocupación y el miedo; de modo que las practicas dialógicas permitieron que la condición existencial en contexto dialógico, diera paso a una comprensión ecológica que facilitó el cambio y la transformación.

Los resultados de este estudio dan cuenta de los procesos particulares de su población, en este sentido no tienen carácter generalizable. En cambio, invitan a la realización de investigaciones que incluyan diversidad de poblaciones, intervenciones y de motivos de consulta.

Me permito terminar adaptando una frase de (Cyrulnik, 2009):

Esta modalidad de intervención breve en grupo se explicó, argumentó y sugirió porque es necesario hablar, dejar hablar y saber escuchar a las heridas y a los heridos de la vida [...], que finalmente somos todos en ese uno que nos habita tan particular y diverso, en ese todo que habitamos...

\section{Referencias}

Anderson, H. (1997). Conversación, lenguaje y posibilidades. Buenos Aires: Amorrortu.

Bateson, G. (1993). Una unidad sagrada: pasos ulteriores hacia una ecología de la mente. Barcelona: Gedisa.

Cyrulnik, B. (2001). La maravilla del dolor. Barcelona: Granica. 
Cyrulnik, B. (2009). Autobiografía de un espantapájaros. Testimonios de resiliencia: el retorno a la vida. Barcelona: Gedisa.

Foucault, M. (1999). Estética, ética y hermenéutica. Buenos Aires: Paidós Ibérica.

Goolishian, H., y Anderson H. (2002). Narrative y Self. Algunos dilemas posmodernos. En Schitman, D. (comp.). Nuevos paradigmas, cultura y subjetividad. Buenos Aires: Paidós.

Hernández, A. (2004). Psicoterapia sistémica breve. La construcción del cambio con individuos, parejas y familias. Bogotá: Códice.

Maturana, H. (1996). El sentido de lo humano. Santiago de Chile: Dolmen.

Miermont, J. (1987). Quelques questions poseès aux models. París: Payot.

Morin, E. (1991). El método V, las ideas. Madrid: Cátedra.

O’Halon, B. (1999). Desarrollar posibilidades. Barcelona: Paidós.

Packman, M. (1995). Redes: una metáfora para una práctica de intervención social. En El lenguaje de los vínculos. Buenos Aires: Paidós.
Sabino, C. (1978). El proceso de investigación. Bogotá: Cid.

Seikkula, J., y Shotter, J. (2010). Diálogos abiertos y prácticas responsivas en crisis extremas. Seminario Sistemas Humanos. Bogotá.

Seikkula, J., y Trimble, D. (2005, diciembre). Healing elements of therapeutic conversation: dialogue as an embodiment of love. Family Process, 44(4), 461-475.

Schnitman, D. F. (1995). Nuevos paradigmas: cultura y subjetividad. Barcelona: Paidós.

Shotter, J. (2011). En el campo de lo dialógico: comprensiones corporales en el ejercicio de las prácticas. Seminario Sistemas Humanos, 8, 9, y 10 de noviembre de 2010. Bogotá.

Watzlawick, P., Beavin, J., y Jackson, D. (1993). Teoría de la comunicación humana. Barcelona: Herder.

White, M., y Epson, D. (1993). Medios narrativos para fines terapéuticos. Barcelona: Paidós. 